\title{
A Collaboration Maturity Model: Development and Exploratory Application
}

\author{
Imed Boughzala ${ }^{1}$, Gert-Jan de Vreede ${ }^{2,3}$ \\ ${ }^{1}$ Telecom Business School \\ Institut TELECOM, France \\ ${ }^{2}$ The Center for Collaboration Science \\ ${ }^{3}$ Systems Engineering Department \\ University of Nebraska at Omaha Delft University of Technology \\ imed.boughzala@it-sudparis.eu, gdevreede@unomaha.edu
}

\begin{abstract}
This paper presents a Collaboration Maturity Model (Col-MM) to assess an organization's team collaboration quality. The Col-MM is intended to be sufficiently generic to be applied to any type of collaboration and useable by practitioners for conducting self-assessments. The Col-MM was developed during a series of Focus Group meetings with professional collaboration experts. The model was piloted and subsequently applied in the automotive industry. This paper reports on the development and first field application of the Col-MM. The paper further serves as a starting point for future research in this area.
\end{abstract}

\section{Introduction}

In today's era of globalization and a knowledge-based economy, organizations have to survive and thrive in a socio-economic environment that is increasingly more unstable and competitive. To meet this challenge, it is critical for organizations to achieve successful collaboration within and among organizational teams and with partners to ensure their organizational performance and competitive advantage $[6,15]$. Indeed, trends like globalization and increased product and service complexity have pushed organizations to use more distributed, cross-disciplinary, virtual teams [5]. In this context, the quality of collaboration directly affects the quality of an organization's outcomes $[1,22]$. This means that the disposition and capabilities of an organization's work force will directly affect organizational performance and thus organizational productivity and profitability [15].

It is important for modern organizations to assess and understand the quality of the collaboration of their teams. Such an understanding can help to identify measures to improve collaboration and therefore lead to improvements in organizational performance. Several researchers have proposed models and approaches to assess either collaboration processes [e.g. 10, 31] or collaboration technology [e.g. 8, 19, 30]. One way to assess the overall collaboration performance of teams is through a maturity model. Maturity models have been used extensively in quality assurance for product development [13]. One of the best-known maturity models is the software Capability Maturity Model (CMM) [29]. Using these types of models as assessment instruments enables organizations to measure at certain intervals and to see trends evolve. Maturity models are not intended to provide rigorous metrics but to help to identify best practices and trouble spots, and to stimulate discussion among practitioners to initiate activities for continuous improvement [12, 32].

Few efforts have been reported on using maturity models to assess collaboration. Lebrun et al.'s [23] model defined maturity levels of concurrent engineering in a virtual company. Their model emphasizes the management of new products and processes in temporary collaborative projects. Fraser et al.'s [12] model is intended to apply to all product development activities; it is not restricted to software products. The model by Ramasubbu et al. [32] focuses on distributed software development. It represents an effort to fill the limitations of models like CMM by introducing several dimensions related to collaboration. Although these models present interesting perspectives on collaboration maturity, few actual applications have been reported. Other limitations include that the models' applicability is limited to certain domains or that they cover just a few phases of the project life cycle $[9,16]$. This paper aims to address this gap. It addresses the following research questions: How can we determine the maturity level of collaboration in organizational teams? What characteristics describe a team's collaboration maturity? What steps need to be taken to measure a team's collaboration maturity?

In this paper, we present the Collaboration Maturity Model (Col-MM) that was developed in cooperation with a Focus Group consisting of professional collaboration experts. The Col-MM is intended to be sufficiently generic to be applied to any type of collaboration and useable by practitioners for conducting self-assessments. We report on the development of the initial version of the Col-MM and its first application in an exploratory field study.

The remainder of this paper is structured as follows. We first summarize research on maturity models. Next, we introduce our research approach to 
develop the Col-MM. Then, we report on the exploratory application and evaluation of the Col-MM in a study in the automotive industry. Last, we present the implications for research and practice, followed by our conclusions which summarize the limitations of this study and present future research directions.

\section{Background}

\subsection{Maturity models}

Maturity literally means 'ripeness'. It describes the transition from an initial to a more advanced state, possibly through a number of intermediate states [13]. Paulk et al. [29, p.21] define process maturity as "the extent to which a process is explicitly defined, managed, measured, and continuously improved". The evolutionary nature of improvement is also emphasized by Shapiro [34, p.147]: "Some do it faster than others and with fewer detours, but fast or slow, every company that gets to world class must evolve through these stages to get there. There are no shortcuts". The fundamental underlying assumption of maturity models is that a higher level of maturity will result in higher performance. Maturity models reflect the degree to which key processes or activities are defined, managed and executed effectively. They typically describe the characteristics of an activity at a number of different levels of performance [12]. "At the lowest level, the performance of an activity may be rather ad hoc or depend on the initiative of an individual, so that the outcome is unlikely to be predictable or reproducible. As the level increases, activities are performed more systematically and are well defined and managed. At the highest level, 'best practices' are adopted where appropriate and are subject to a continuous improvement process” [12, p.1500).

Approaches to determine process or capability maturity are increasingly applied to various aspects of product development, both as an assessment instrument and as part of an improvement framework [11]. Most maturity models define an organization's typical behavior for several key processes or activities at various levels of 'maturity' [12]. Even though maturity models are declarative, i.e. based on self-reports, they provide an instantaneous snapshot of a situation and a framework for defining and prioritizing improvement measures. Key strengths of maturity models include:

- They are simple to use and often require simple quantitative analysis;

- $\quad$ They can be applied from both functional and cross-functional perspectives;

- They provide opportunities for consensus and team building around a common language and a shared understanding and perception;
- They can be performed by external auditors or through self-assessment.

One of the earliest maturity models is Crosby's Quality Management Maturity Grid (QMMG) [7], which was developed to evaluate the status and evolution of a firm's approach to quality management. Subsequently, others maturity models have been proposed for a range of activities including quality assurance, software development [29], supplier relationships [25], innovation [4], product design [14], R\&D effectiveness [27], product reliability [33], and knowledge management [21]. The best known models are the CMM for software engineering (based on the Process Maturity Framework of Watts Humphrey, quoted in [29]), developed at the Software Engineering Institute (SEI), and the ISO 9001 standard. Both share a common concern with quality and process management. Unlike the other maturity models cited above, CMM is a more extensive framework in which each maturity level contains a number of key process areas (KPAs) containing common features and key practices to achieve stated goals. A number of studies of the software CMM have shown links between maturity and software quality [e.g. 17, 18]. This model is widely used in the software industry as part of quality certification.

Several maturity models aim at clearly identifying the organizational competences associated with best practices [13]. In practice, however, many maturity models are intended to be part of an improvement process, and not primarily as absolute measures of performance [13]. Few maturity models have been validated in the way of performance assessment. An exception is Dooley et al.'s [11] study that demonstrated a positive correlation between New Product Development (NPD) process maturity and outcome.

\subsection{Collaboration maturity models}

The word "collaboration" derives from the Latin words com and laborare which mean "to work together". Researchers have defined collaboration in different ways. For example, Levan [24] states that collaboration is a social phenomenon that involves several individuals when the action of only one does not achieve the expected result. Briggs and colleagues define collaboration in terms of efforts and purpose: "Collaboration is making a joint effort toward a group goal” [3, p.122]. In the context of this study, we define collaboration as a process in which two or more agents (individuals or organizations) share resources and skills to solve problems so that they can jointly accomplish one or more activities. During this process, the agents communicate with each other to coordinate their tasks. Based on this definition of collaboration, 
we define collaboration maturity as a team's current maximum capability to collaborate where team members effectively communicate, reach shared understanding, and adjust their tasks and behaviors to produce high quality outcomes.

Recently, a number of maturity models related to collaboration have been proposed:

1. The Fast Reactive Extended Enterprise-Capability Assessment Framework (FREE-CAF) model [23]. The purpose of this model is to define the maturity level of concurrent engineering in a virtual company. It emphasizes the organization and management of new products and processes in temporary collaborative projects in two or more organizations. The model distinguishes four maturity levels (repeatable, defined, managed, and optimizing). At each level, competences are defined for each Key Process Area (KPA). The latter include inherent practices of the virtual organization, the partners, or both. The FREE-CAF model is interesting as it incorporates a wide range of KPAs, yet it is limited in that it covers only the initial phases (design and engineering) of the project life cycle.

2. The Collaboration Maturity Grid (CMG) was developed at Cambridge University to examine collaborative maturity in firms that outsource a significant amount of design or development activity to a third party [12]. The model aims to provide guidance to managers involved in collaborative projects. This model applies to the development of products in general and is not restricted to the domain of software. A limitation is that it mainly focuses on the organizational-level collaboration between companies rather than on the team-level work practices and tools.

3. The model by Ramasubbu and colleagues [32] was developed at SAP AG in response to limitations with CMM. It emphasizes the dimensions of dispersion and collaboration of development teams. Both CMM and ISO 9001 do not address the key processes required to develop or evaluate distributed products such as establishing mutual knowledge and managing geographically dispersed social networks. A strength of the model is that it addresses four concepts that are central to distributed software development [28]: collaboration readiness, common ground, coupling in work, and technology readiness.

Each of the above collaboration maturity models is founded on the assumption that the quality of a product is related to the quality of the collaboration process. The value of these models is that they emphasize and raise awareness about collaboration maturity in an organizational setting. Yet, limitations exist. First, few applications have been reported and reported ones have not been validated empirically [9, 16]. Second, their application is specific for only certain types of collaboration (e.g. inter-organizational or distributed projects), for certain application domains, or for certain project life cycle phases. Third, most models are descriptive in nature and do not propose solutions. Finally, little is known about whether the use of these models leads to actual performance improvements.

The main objective of the study reported in this paper is to present the blueprint for a new collaboration maturity model that addresses some of the limitations described above. This model aims to be generic for all types of collaboration and can be used to assess the collaboration maturity of a given team holistically. Further, it supports the development of recommendations to improve the quality of collaboration outcomes and thus collaboration performance.

\section{Method}

The present research is based on the Design Science approach. Design science research tries to meet the identified business needs through the building and evaluation of artifacts [20]. These artifacts are built to address unsolved problems, and are evaluated with respect to the utility they provided in solving these problems. This approach is well suitable for our research since it aims to develop a model to assess the collaboration maturity in an organization, the Collaboration Maturity Model (Col-MM). Further, our research aims to evaluate and demonstrate the model's practical feasibility and utility through pilot and field studies (observational methods according to Hevner et al.’s [20] Design Evaluation Framework).

Constructs, models, methods, and instantiations are the four design artifacts produced by designscience research in IS [26]. For our research on ColMM these artifacts would be represented as follows:

- Constructs: The Col-MM structure that describes the collaboration areas of concerns (topics) and their related criteria.

- Model: The Col-MM questionnaire that includes questions, levels of rating and mathematical formulas for analysis.

- Method: The Col-MM method that (a) defines the steps and provides guidance on how to run the ColMM questionnaire in the field, and (b) supports the development of recommendations.

- Instantiation: The Col-MM tool which is a customized MS Excel application that represents the implementation of the above artifacts, and enables the execution of a concrete assessment.

The development and application of the Col-MM is summarized in Figure 1. First, based on the literature we identified the main previous research in the area of maturity models in general and of collaborationoriented ones in particular. Second, to maximize the 
proposed maturity model's relevance and practical applicability, collaboration experts were involved from the early stages of the development of Col-MM artifacts and during the pilot studies in the field. Third, the model was field-tested in the automotive industry to validate the artifacts. Further field studies should be executed to further evolve the Col-MM artifacts.

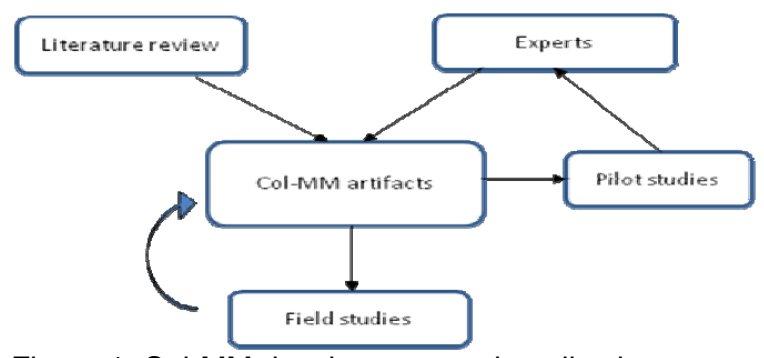

Figure 1: Col-MM development and application steps.

\section{The Collaboration Maturity Model}

\subsection{Development of the Col-MM}

The Col-MM was developed in cooperation with a Focus Group consisting of professional collaboration experts. These experts regularly met in the context of a business association (a) to share best practices on methods, techniques and tools, (b) to get their peer's feedbacks on case studies, and (c) to attend special presentations on the latest trends in the collaboration and knowledge management area. The involvement of the experts group enabled us, in the words of Hevner et al.'s IS research Framework [20, p.80], to combine relevance and rigor by meeting a business need with applicable knowledge and so to maximize the resulting artifacts' relevance and applicability.

The Focus Group meetings took place over the course of 20 months. The goal of these meetings was threefold. First, to build a generic collaboration maturity model for the holistic assessment of teams. Second, to apply the model in practice through pilot and field studies. Finally, to provide guidelines and a tool to enable practitioners for conducting selfassessments with the Col-MM.

Participants. The Focus Group experts included 15 Chief Knowledge Officers (CKOs) working for different companies of different sizes (ranging from 500 - 1,000 to 100,000-200,000 employees; including 10 multinational firms) in different sectors (including Automotive, Software, Audiovisual, Civil engineering, and Telecommunications). The participants held at least a master-level degree (four held a PhD). They had at least 15-19 years of work experience, with $50 \%$ of them 5-9 years as a CKO. The average age was 48 years. $73 \%$ were male. All participants were French.
Focus Group Process. The development of the Col-MM took almost two years. The Focus Group process consisted of three-hour long monthly meetings, facilitated by one of the researchers. Seven working meetings were used to work on the Col-MM artifacts, five meetings for participants' feedbacks on pilot studies, and six hosted external thematic presentations related to maturity models and collaboration from professional and research perspectives. The seven working meetings covered the following steps in the Focus Group process:

1. The first step consisted of the generation of the antecedents to collaboration performance. Following this meeting, three thematic presentations were planned on the topic.

2. The second step was to generate the requirements, analysis levels, and topics of analysis for a useful collaboration maturity model. Two thematic presentations were provided after this meeting: one on capability models including CMM and one on a literature review of collaboration maturity models. The participants expressed the following requirements for the Col-MM:

- Resource efficient: The Col-MM should be fast to complete.

- Rich data: The Col-MM should report different perspectives from the workplace, using both quantitative and qualitative data.

- Limited need for further advanced data analysis: The supporting tool should provide integrated support for results interpretation.

- Self-assessment: Practitioners should be able to apply the Col-MM themselves.

- Constructive learning: The Col-MM should promote team building and organizational learning rather than control and sanction.

3. In the third step constructs for the Col-MM were generated in terms of criteria and levels of assessment. This meeting was followed by one thematic presentation on data analysis techniques.

4. The fourth step focused on the development of the Col-MM questionnaire in terms of items, rating levels, average calculations, and weightings.

5. The fifth step addressed the Col-MM method in terms of how to scope a Col-MM project, how to choose respondents and how to run the questionnaire in practice. Following this meeting, the Col-MM was tested in three pilot studies that were organized in the companies of three of the participants. As a result, three feedback presentations and group discussions were planned.

6. In the sixth step the use of the Col-MM method was evaluated. Several adjustments and improvements were proposed and discussed in this meeting. After 
that, the Col-MM Excel tool was designed (by a third party) and two other pilot studies were presented.

7. In the final step, both the Col-MM method and tool were validated by the focus group in the presence of the CEO of a large company that was interested in hosting a Col-MM field study.

\subsection{The Col-MM}

The Col-MM aims to assess the maturity of a given team holistically. It supports the development of recommendations in form of an action plan to reach improved collaboration performance. Its applicability is not limited to a particular form of collaboration and the model can be used for different settings (e.g. project teams, organizational teams, cross functional/organizational teams, inter-organizational team, or communities of practice).

Structure. Col-MM distinguishes between four maturity levels: Ad-hoc, Exploring, Managing and Optimizing. At the Ad-hoc level, teams are collaboratively immature. Individuals have many difficulties to communicate effectively, to reach shared understanding, and to adjust their tasks and behaviors to produce high quality outcomes together. At the Exploring level, teams are well aware of their weaknesses in terms of collaboration maturity. Individuals try work together to produce valuable outcomes, but are faced with many collaborative challenges. Some initiatives to address these are attempted but without major impacts. At the Managing level, the collaboration maturity of teams is quite good but there still is room for improvement. In general, individuals are able to produce collaborative outcomes of good quality. At the Optimizing level, teams are collaboratively mature. Individuals work together optimally and are able to accomplish high quality collaborative outcomes.

Unlike the other maturity models discussed earlier, Col-MM explores the maturity of a given team holistically from different perspectives related to collaboration. The following perspectives, or areas of concerns, were considered essential by the participants in the Focus Group meetings:

- Collaboration characteristics: This covers descriptive features and attributes of the collaboration.

- Collaboration management: This covers the way in which collaboration processes and activities are managed.

- Collaboration process: This covers how actors perform collaboration on a regular basis.

- Information and knowledge integration: This covers how actors manage the information and knowledge required for productive collaboration.
For each area of concern, a number of criteria were defined (see Table 1). These criteria represent the topics for the Col-MM questionnaire. Each criterion is represented by an item that is evaluated on a 4-level scale. To support the respondents, the levels of each criterion are described briefly, with examples wherever possible. An example of a criterion item is provided in Figure 2. Respondents are allowed to provide scores such as “0.5”, “1.5”, “2.5”, and “3.5”. When a respondent cannot answer, no score is recorded. The more the criteria are rated at 4 by respondents, the higher the maturity of collaboration is considered to be.

Table 1: Col-MM areas of concern and criteria.

\begin{tabular}{|c|c|}
\hline Area of concern & Criteria \\
\hline \multirow{8}{*}{$\begin{array}{l}\text { Collaboration } \\
\text { Characteristics }\end{array}$} & 1. $\quad$ Collaboration object \\
\hline & 2. $\quad$ Collaboration depth \\
\hline & 3. $\quad$ Working mode \\
\hline & \begin{tabular}{|ll}
4. & Interaction intensity \\
\end{tabular} \\
\hline & 5. Collaboration forms \\
\hline & 6. $\quad$ Formalization of relationships \\
\hline & $\begin{array}{ll}\text { 7. } & \begin{array}{l}\text { Commitment and availability of } \\
\text { individuals }\end{array} \\
\end{array}$ \\
\hline & 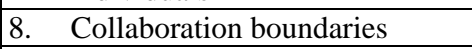 \\
\hline \multirow{6}{*}{$\begin{array}{l}\text { Collaboration } \\
\text { Management }\end{array}$} & 9. $\quad$ Collaboration goal \\
\hline & 10. Management style \\
\hline & 11. Decision-making \\
\hline & 12. Leadership endorsement \\
\hline & 13. $\quad$ Rewarding \\
\hline & 14. Collaboration progress \\
\hline \multirow{4}{*}{$\begin{array}{l}\text { Collaboration } \\
\text { Process }\end{array}$} & 15. Collaboration framework \\
\hline & 16. Resources sharing \\
\hline & 17. Awareness \\
\hline & 18. Conflict management \\
\hline \multirow{6}{*}{$\begin{array}{l}\text { Information and } \\
\text { Knowledge } \\
\text { Integration }\end{array}$} & 19. Information collection \\
\hline & 20. Information structuring \\
\hline & 21. Information access \\
\hline & 22. Knowledge validation \\
\hline & 23. Knowledge reusing \\
\hline & 24. Knowledge creation \\
\hline
\end{tabular}

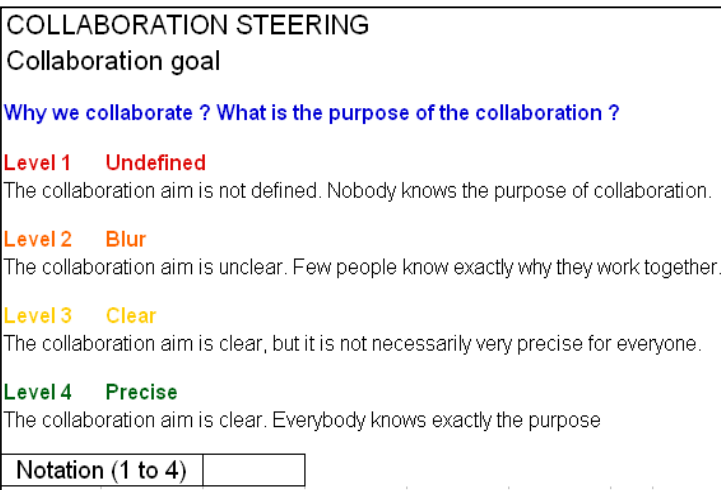

Figure 2: Example of criterion in Col-MM. 


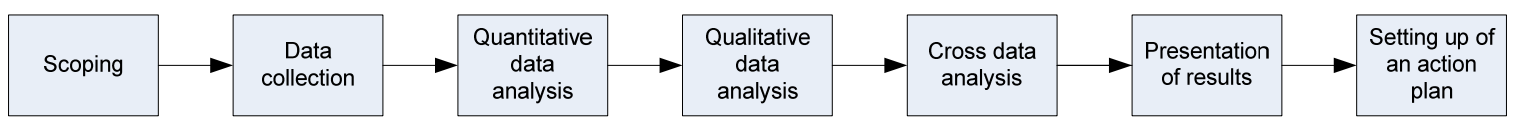

Figure 3. The seven steps in the Col-MM method.

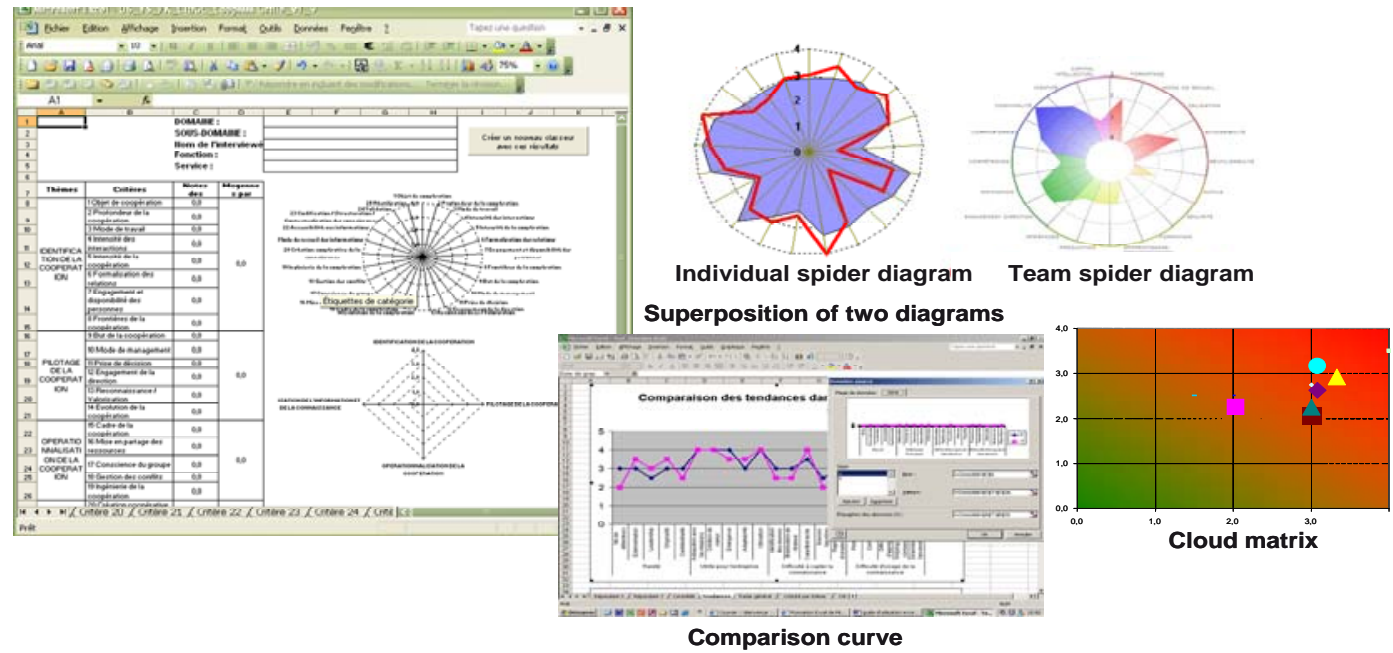

Figure 4. The Col-MM tool data collection and analysis.

In essence, the Col-MM is structured as a library of criteria. Sometimes, not all criteria are relevant. So, an organization can decide which criteria fit better with a particular context. It can also decide to expand the set of criteria. Also, for some organizations certain criteria may be more important than others. In such situations, it is possible to assign different weights to the criteria.

Process. The Col-MM method defines the steps to perform the analysis. Figure 3 summarizes the seven main steps in the method. At the scoping step, the purpose of the Col-MM analysis is defined according to the organizational context and the business strategy. The boundaries of the analysis (i.e. organizational scope) are defined before starting. The reasons for performing the assessment should be communicated to all concerned individuals and teams. It is important to present this as a holistic team assessment to improve overall organizational performance, rather than as an individual retrospective evaluation.

The data collection is performed through individual and/or collective interviews based on the Col-MM questionnaire (quantitative data). The selection of individuals should be representative of the target team. During interviews, qualitative observations should be collected to enrich the analysis and gain a deeper understanding of any perception differences that may exist. After the data collection, a first quantitative data analysis is performed using the ColMM tool (described below). This analysis presents individual perceptions about the collaboration maturity of the team. It also helps to identify critical perception differences concerning the different criteria. The qualitative data analysis (using a content or thematic analysis based on the interview statements) helps to get a more in-depth understanding of these perception differences for each criterion or group of criteria (area of concern). Follow-up discussions and consensus building efforts could be carried out for relevant scores, in order to settle on an acceptable assessment. The cross analysis may yield additional interpretations by combining criteria for specific measurements of capabilities according the goal of the assessment, such as value creation (criteria 4, 23, 24) or organizational learning (criteria 10, 16, 19, 20, 21, 22, 23, 24).

The analysis results can be presented in various formats using the Col-MM tool (Figure 4). Individual spider diagrams can be made individually for all criteria scores or grouped by topic. Superposition of individual spider diagrams can show the rating gaps on individual criteria or topics. To identify criteria for which it is necessary to collect additional information comparison curves can be prepared that visualize perception differences between different respondents regarding the same criterion. Team spider diagrams of all criteria scores individually or grouped by topic can represent the collective perception of the collaboration maturity of the team. Finally, cloud matrices can show combinations of criteria.

The last step of the Col-MM method concerns the definition of an action plan. It helps in the framing of concrete recommendations in terms of actions to improve collaboration performance and the quality of collaboration outcomes. Such actions may involve a variety of initiatives, for example, the clarification of 
the strategic corporate objectives, training on facilitation techniques (e.g. for brainstorming), invited seminars on collaboration technologies, coaching/tutoring, the development of a shared knowledge repository, group communication training.

Col-MM tool. The Col-MM Excel application allowed quantitative and qualitative data collection during the interviews and to analyze quantitative data automatically. It provides different presentations of results and the results' report generation.

\section{The Application of Col-MM: A field study in the Automotive industry}

A large multinational automotive firm had a desire to assess the collaboration performance of some of their distributed virtual teams. This company has previously established a new organizational matrix structure, based on the "management by project" principle. To assess the 'fit' of this new structure in the context of a recent merger-acquisition and to see if all the constituent brands work as a one single group, the company decided to assess the overall organizational performance in terms of synergy between the different sites and brands, productivity, quality of the products, and the balance between product diversity and process complexity. The collaboration maturity assessment was part of this larger organizational performance assessment.

As a first step it was decided to apply the Col-MM to measure the collaboration maturity of one virtual team distributed over two European countries (two sites) with different cultures, work habits, and management styles. This team was in charge of the "Engine After Treatment System" (EATS) that was part of a larger development project of a new diesel engine that was taking place under the responsibility of a business unit distributed over three countries. The leading site in this project will be referred to as site A.

\subsection{Field study steps}

The field study followed the Col-MM method steps during four months. The objectives were to check:

- If the organization had adequate capabilities to effectively support high quality collaboration.

- If collaboration technologies were provided and if they were effectively used.

- If there were critical issues related to cultural differences (national, organizational, technological, etc), given the merger-acquisition context.

The selection of respondents was done with the assistance of the firm' CKO according to criteria such as job position, responsibility, process step intervention, working experience, etc. All respondents had similar levels of education (MSc degree) but from different engineering fields: mechanical, electronic, electric, and industrial. The Col-MM questionnaire was sent to the respondents before the meeting with an introduction to the company's Col-MM objectives. Anonymity and confidentiality of the treatment of the responses were formally assured.

Nine individual interviews were conducted faceto-face in the respondents' native language in the two European countries ( 3 in the site A and 6 in the site B). Next, two collective interviews (one for each site) were conducted to examine perception gaps on some criteria. Each interview lasted about 90 minutes. During the interviews the Col-MM tool was used for data collection. All interviews were recorded for further qualitative data analysis.

After the quantitative analysis with the Col-MM tool, the qualitative analysis of specific statements, and cross data analysis, a first report was sent to the respondents to solicit corrections before the final report was prepared for the company's top management.

\subsection{Findings}

The findings were reported as a discussion of the different perceptions related to the Col-MM criteria. Examples of findings reported to top management according to the Col-MM areas of concern include:

- Collaboration characteristics: We found similar understandings of collaboration goals and team members' commitment for both sites.

- Collaboration management: We noticed different perceptions between the two sites with respect to management style and decision-making (hierarchical management vs. consensual management). Site B respondents felt unfairly rewarded compared to site A. They felt that because site A had the project lead, its employees always had an advantage.

- Collaboration process: We noticed that site B respondents had less awareness about different collaboration approaches to enhance the team performance. Because of their positions and responsibilities in the process, they focused more on their individual contribution to the overall process rather than on developing collaborative relationships. We also noticed differences in terms of conflict management by the leadership in each site: Conflict management in the site $A$ is based on consensus while in site B it is based on hierarchical decision making and negotiation.

- Information and Knowledge integration: we found different perceptions regarding information access. For site A respondents, access to information is not as well organized as well as they would wish. 
Information is very distributed and access should be simplified. We found the consistent perceptions between the two sites regarding collaborative knowledge creation; both sites felt this process was well organized.

Through the qualitative data analysis, we found that some culture differences between sites appeared to be related more the organizational culture rather than to the national culture. For example, the balance between private and professional life appeared to be different. Also, there was a different brand identity: Site B respondents felt they were still belonging to their original brand (i.e. from before the merger) than to the group of brands. We also found different work attitudes: In site $\mathrm{B}$ respondents were more reactive compared to the respondents in site A being more proactive. According to some respondents, this is because of their position in the project. Possible explanations could be related to their contracts type (tenure status) and social protection.

The findings further included that collaboration appeared to be mainly based on "individuals' goodwill”, for example related to resource sharing and knowledge management. Also, the team turned out not to be as collaboratively mature as was expected - they were at the exploring level. Because of the asymmetric collaboration awareness between the two sites, their collaboration was mostly of a coordination nature. This makes it difficult to further improve the quality of their outcomes. Finally, the new matrix structure did not resolve all problems with respect to the imbalance between responsibility and authority. The final report made various recommendations, including:

1. Make collaboration a clear strategic goal in all project management initiatives.

2. Re-think the management of collaboration and provide training for managers.

3. Nominate full-time facilitators for collaboration.

4. Take diversity aspects related to culture into account. 5. Make explicit recognitions for the contributions of every actor toward effective collaboration.

After six months, we learned that three of the suggested recommendations were followed up with concrete actions. The first recommendation was clearly mentioned in the company's project management standard. Following the third recommendation, one full time collaboration facilitator was assigned to each business unit. Following the fifth recommendation, a 'collaboration capability' criterion was added to the annual individual performance assessment.

\subsection{Reflection on the application of Col-MM}

After applying the Col-MM steps during this field study, we gathered experiences and feedback regarding the appropriateness and usefulness of Col-MM through semi-structured interviews, the results of which were analyzed using an open coding approach to uncover common themes. According to the respondents, the Col$\mathrm{MM}$ analysis was useful and correctly represented their perceptions. It focused on real collaboration problems and allowed traditionally 'unspoken issues' to surface. They were also satisfied with the feedback provided to top management and the subsequent actions that were taken related to the assessment's recommendations. According to the operational managers, the results were relevant. Further, most of them felt able to reuse the ColMM by themselves in the future. According to the top managers, the study was satisfactory in terms of results and recommendations, as they confirmed and reinforced some of their own perceptions. This allowed them, for example, to focus more on the organizational culture than on national culture and to understand the problems related to the project-based new organizational structure.

We also received feedback and recommendations from the respondents on the Col-MM questionnaire such as the possibility to review some criteria and questions. They respondents stated that some criteria were a little difficult to understand. Also, the nuances between levels of responses were sometimes subjective or difficult to distinguish. In addition, they proposed to add some criteria such as culture, work experience, and practice diversity, and to rename some areas of concern such as "collaboration readiness" instead of "collaboration characteristics". Finally, they suggested putting a stronger focus on collaboration technology rather than on information and knowledge integration. Interestingly, but not surprising given the CKOs' interests, this was contrary to the wishes expressed by the focus group. However, since the Col-MM is developed as a library of criteria, the review of the Col-MM structure according to a specific context is possible and therefore suggestions can be easily accommodated. In terms of execution, most respondents expressed that they preferred the use of collective rather than individual interviews as this would enable a faster application of the Col-MM process.

Based on the experiences and feedback from this field study, we make the following observations regarding the extent to which Col-MM meets its initial critical requirements:

- Resource efficient: A total of 36 hours were spent: 1.5 hours for the assessment preparation, 16.5 hours for the interviews (engineering profile), 3 hours for the CKO profile, 3 hours for the top management profile and 12 hours for the analysis and report preparation. We feel that this is a comparatively modest and reasonable effort in terms of resources spent. 
- Rich data: The combined use of quantitative and qualitative data analysis resulted in richer finding. We felt that qualitative observations enabled us to better uncover and interpret the various points of views expressed by the respondents through the Col-MM questionnaire.

- Limited need for further advanced data analysis: The analysis needs in the field study were limited and the Col-MM tool provided sufficient support (among others the report generation).

- Self-assessment: The operational managers expressed confidence that they could perform future applications of the Col-MM themselves.

- Constructive learning: Respondent feedback shows that if the Col-MM study is carefully communicated, participation can be effective and generate discussions on real problems that further facilitate the acceptance of proposed solutions. For this, anonymity and confidentiality seem to be crucial.

\section{Discussion}

We followed the seven guidelines for Design Science [20]. In order to produce new artifacts (Col-MM structure, questionnaire, method, and tool) to be added as applicable knowledge to the knowledge base (see framework in [20, p.80]), we developed a purposeful method and application (Guideline 1: Design as an Artifact) showing step by step how to solve a specific problem related to the holistic assessment of the collaboration maturity of a team. This problem meets a clear business need as expressed by professionals as a means to reach better productivity and performance (Guideline 2: Problem relevance). A total of five pilot case studies in five companies and one field application in a multinational firm using observational methods were executed to evaluate the appropriateness and usefulness of the Col-MM, with the active contribution from a Focus Group of experts (Guideline 3: Design Evaluation). Our literature review showed that there is limited guidance available to determine and assess collaboration maturity in teams and organizations, while concerned experts confirmed a clear business need (Guideline 4: Research contributions). The development was rigorously defined (Guideline 5: Research Rigor) using a combination of research methods including a literature review, an expert focus group, and empirical field studies (Guideline 6: Design as a search process). Finally, the results of our study are and will be communicated in two steps (Guideline 7: Communication of Research): First, the method and initial experiences are presented through publications to other researchers who, we hope, will consolidate and extend the Col-MM method and application, and to practitioners who could apply the method and provide feedback and recommendations for its future enhancement. Second, after further study of the method and its application in various contexts (other field studies), top managers could decide to use it as a strategic instrument to improve their teams' performance.

\section{Conclusions}

This paper proposes a first version of a collaboration maturity model, Col-MM, to assist in the assessment of teams' collaborative performance. The Col-MM was developed using a constructivist approach (Design Science) to meet a real business need as expressed by 15 CKOs and others experts that are regularly confronted with such challenges. Our contribution is both theoretical and practical as we propose a model, an application method, a supporting tool, and an empirical evaluation of their application. Our experiences show that the Col-MM can be applied in a resource-efficient fashion and yields results that are useful for organizations.

Nevertheless, there are limitations related to this study. First, our empirical evidence is based on a single field study. Further field studies have to be executed to expand the evaluation of the Col-MM artifacts and to further enhance the Col-MM. Particular care will have to be taken to ensure that Col-MM can take into account all levels of collaboration and all collaboration processes in organizations in different settings. This cannot be achieved by just expanding the number of criteria as this will overly complicate the use of the model. Second, at this stage, the Col-MM cannot yet be used to investigate a correlation between collaboration maturity levels and organizational/team performance. However, it provides a first step into this direction. The results should be of interest to academic researchers and information systems practitioners interested in team collaboration and project management. Our research contributes to collaboration literature, theory and practice through the development of Col-MM artifacts that provide evidence of proof of value and proof of use in the field.

We recommend several directions for future research to enhance the current version of Col-MM. First, the model has to be applied in different types of organizations for different types of teams. The experiences from these applications will assist in the further development and evaluation of the Col-MM artifacts. Second, the weighting of criteria, not detailed in this paper, should be further explored and correlated with the four levels of maturity. Third, organizational and team performance measures have to be developed 
to enable an analysis of the relationship between collaboration maturity and organizational productivity. Finally, from a Behavioral Science perspective, some further confirmatory studies should be performed using Structural Equation Modeling [2] to validate the correlation between the Col-MM constructs and team or organizational performance.

\section{References}

[1] Banker, R., Bardhan, I., Asdemir, O. (2006). Understanding the impact of collaboration software on product design and development, Information Systems Research, Vol. 17, No.4.

[2] Bollen K. A. (1989). Structural Equations with Latent Variables. New York: Wiley.

[3] Briggs, R. O., Kolfschoten, G. L., Vreede, G. J. de, \& Dean, D. L. (2006). Defining Key Concepts for Collaboration Engineering, Proceedings of the 12th Americas Conference on Information Systems, 121-128.

[4] Chiesa, V., Coughlan, P. and Voss, C. (1996). Development of a technical innovation audit. J. Product Innovation Management, 13(2), 105-136.

[5] Chudoba K. M., Wynn E., Lu M., Watson-Manheim M. B. (2005). How virtual are we? Measuring virtuality and understanding its impact in a global organization, Information Systems Journal, 15, 279-306

[6] Clark, K., T. Fujimoto. (1991). Product development performance: Strategy, organization and management in the world auto industry. Harvard Business School Press, Boston, MA.

[7] Crosby, P. B. (1979). Quality is Free, McGraw-Hill, New York.

[8] Damianos L., Hirschman L., et al. (1999). Evaluation for Collaborative Systems, ACM Computing Surveys, 31, 2.

[9] Daoudi J., Bourgault M. (2007) What Do We Know About Collaborative Maturity? 4th Annual Sprott Doctoral Symposium, April 19-20.

[10] Den Hengst M., Kolfschoten G., Dean D., Chakrapani A. (2006). Assessing the quality of collaborative processes. Proceeding of the 39th Hawaii International Conference on System Sciences, IEEE Computer Society.

[11] Dooley, K., Subra, A. \& Anderson, J. (2001). Maturity and its Impact on New Product Development Project Performance, Research in Engineering Design, 13:30-29.

[12] Fraser, P., Farrukh, C. \& Gregory, M., (2003). Managing product development collaborations - A process maturity approach, Proceedings of the Institution of Mechanical Engineers, 217, 11, 1499-1519.

[13] Fraser, P., Moultrie, J. \& Gregory, M., (2002). The use of maturity models / grids as a tool in assessing product development capability, IEEE International Engineering Management Conference, Cambridge, August 19-20.

[14] Fraser, P., Moultrie, J. \& Holdway, R. (2001). Exploratory studies of a proposed design maturity model. 8th Intern. Product Development Management Conference, University of Twente, Holland, June 11-12.

[15] Frost \& Sullivan (2007). Meetings Around the World: The Impact of Collaboration on Business Performance. White paper available at http://newscenter.verizon.comkit/collaboration/MAW_WP.pdf. (Accessed: 4/3/2011)
[16] Hain S., Back A. (2009 July). State-of-the-art on maturity models for collaboration, http://web.iwi.unisg.ch/org/iwi/iwi_pub.nsf/0/F4A4973DEFFE7034C1 25765E00502373/\$file/State-of-the-Art 01 2009.pdf

[17] Harter, D., Krishman, M., Slaughter, S. (2000). Effects of Process Maturity on Quality, Cycle Time, and Effort in Software Product Development. Management Science, 46, 451-466.

[18] Herbsleb, J., Zubrow, D. Goldenson D., Hayes W., Paulk M. (1997). Software quality and the capability maturity model, Communications of the ACM, 40, 9, 30-40.

[19] Herskovic V., Pino J., Ochoa S., Antunes P. (2007). Evaluation Methods for Groupware Systems, Groupware: Design, Implementation, and Use, LNCS, Springer, Volume 4715, 328-336.

[20] Hevner A., March S., Park J., \& Ram S. (2004). Design Science in Information Systems Research, MIS Quarterly, 28, 1.

[21] Hsieh, P.J., B. Lin, C. Lin (2009), The Construction and Application of Knowledge Navigator Model (KNM TM). Expert Systems with Applications, 36, 4087-4100.

[22] Jordan, M. H., Field, H. S., \& Armenakis, A. A. (2002). The relationship of group process variables and team performance. Small Group Research, 33, 121-150.

[23] Lebrun, J., Gosset, P., Pallot, M., Roux, P., Gandelot, D., Vannier, M., (1998). Capability Assessment Framework - CAF, Volume 1: Overview, Deliverable No. 132-1, Esprit Project 23286.

[24] Levan, S.K. (2004). Travail collaboratif sur Internet: Concept, méthodes et pratiques des plateaux projet, Paris, France: Vuibert.

[25] Macbeth, D., Ferguson, N. (1994). Partnership sourcing: an integrated supply chain management approach, London: Financial Times: Pitman Publishing.

[26] March, S. T. and Smith, G. (1995). Design and Natural Science Research on Information Technology, Decision Support Systems (15:4), December 1995, pp. 251-266.

[27] McGrath, M. E. (Ed.) (1996). Setting the PACE in Product Development: A Guide to Product and CycleTime Excellence, Butterworth-Heinemann, Oxford.

[28] Olson G.M., Olson J.S., (2000). "Distance Matters", Human Computer Interaction, vol. 15, pp. 139-179.

[29] Paulk, M., Curtis, B., Chrissis, M. Weber, C. (1993). Capability maturity model for software, Version 1.1. Technical Report CMU/SEI-93-TR-024 ESC-TR-93-177.

[30] Pinelle D., Gutwin C. (2003). Task analysis for groupware usability evaluation: Modeling shared-workspace tasks with the mechanics of collaboration, ACM Transactions on Computer-Human Interaction, 10, 4, 281-311.

[31] Pinsonneault A., Kraemer K. (2002). Exploring the Role of Information Technology in Organizational Downsizing: A Tale of Two American Cities. Organization Science, 13, 191-208.

[32] Ramasubbu, N., Krishnan, M., Kompalli, P. (2005). A process maturity framework for managing distributed development, IEEE Software, May/June, p. 80-86.

[33] Sander, P., Brombacher, A. (2000). Analysis of quality information flows in the product creation process of high-volume consumer products, International Journal Of Production Economics 67 (1): 37-52.

[34] Shapiro, A. (1996). Stages in the evolution of the Product Development Process, in McGrath, Michael E. (ed). Setting the PACE in Product Development: a guide to product and cycle-time excellence. Butterworth-Heinemann. 